\title{
The Development and Delivery of a Systematic Veterinary Clinical Skills Education Program at the University of Calgary
}

\section{Emma K. Read ${ }^{1 *}$ and Kent G. Hecker ${ }^{2}$}

${ }^{1}$ Senior Instructor Equine Surgery Chair, Clinical Skills Courses, Faculty of Veterinary Medicine, Department of Veterinary Clinical and Diagnostic Sciences, University of Calgary, 1187785 Street NW, Calgary, Alberta, T3R 1J3, Canada

${ }^{2}$ Assistant Professor Veterinary Medical Education, Faculty of Veterinary Medicine, Department of Veterinary Clinical and Diagnostic Sciences, University of Calgary, 3330 Hospital Drive NW Calgary, Alberta, T3H 4N1, Canada

\begin{abstract}
The University of Calgary Faculty of Veterinary Medicine (UCVM) outcomes based, core-integrated curriculum model emphasizes integration of basic sciences, clinical and professional skills through horizontally integrated courses beginning in the first year of the program. The Clinical Skills course constitutes $20 \%$ of the curriculum for each of the first three years of the program. This article reviews the theoretical foundation, development, delivery and assessment of student performance in the clinical skills course, including creation of essential skill lists, development of clinical simulators, creation of learning sessions, incorporation of sessional instructors from the distributed teaching hospitals, and development of objective structured clinical examination (OSCE) assessments.
\end{abstract}

Keywords: Veterinary education; Clinical skills; Technical skills; Practical skills; OSCE; Essential skills list; Simulation

Abbreviations: OSCE: Objective Structured Clinical Examinations; UCVM: University of Calgary Faculty of Veterinary Medicine; DVLC: Distributed Veterinary Learning Community; DVM: Doctor of Veterinary Medicine; CSB: Clinical Skills Building

\section{Introduction}

Faculty of Veterinary Medicine, University of Calgary (UCVM) is a 4 year DVM program that was established in 2005. The curriculum has been developed as an outcomes-based, core-integrated model, meaning that approximately $60 \%$ of the curriculum is dedicated to basic veterinary sciences courses, and the remainder of the curriculum is assigned to three foundational "super" courses. The final year of the program involves clinical rotations that take place in the distributed veterinary learning community (DVLC) that is comprised of 68 primary care practices and 5 government agencies within the province of Alberta. "Clinical Skills", is one of the foundational courses in the curriculum, and represents a newly developed approach to practical skills training in veterinary medicine. The clinical skills courses are delivered in an 80,000 square-foot, purpose-built building, that includes a clinical simulation laboratory. This paper outlines the genesis and delivery of the clinical skills teaching program including: 1. theoretical foundation, curricular and instructional design phases, 2 . physical infrastructure requirements, 3. personnel requirements, and 4 . assessment of student's clinical skills performance.

\section{Theoretical foundation, curricular and instructional design of the skills program}

One of the challenges in developing a clinical skills program is defining the limitations of what constitutes a "clinical skill" and organizing those skills into a logical course framework. A clinical skill has been described previously as having several essential components including procedural knowledge, declarative knowledge, and clinical reasoning/critical thinking [1]. Procedural knowledge relates to the technical know-how for performing the skill. Declarative knowledge relates to the justification for performing the skill, as well as to the critical information that pertains to the anatomy and physiology involved. Clinical reasoning and critical thinking encompass diagnostic reasoning, clinical decision making and any pertinent evidence basis
[1]. A clinical skill taught without these knowledge components is simply mechanical performance, and has limited diagnostic value since it cannot be adapted to new patients or novel situations that will arise in clinical practice [1].

Schools are continually challenged to teach veterinary students the clinical skills they will require upon graduation [2]. To address this challenge, the UCVM clinical skills program was purpose-designed to teach clinical skills using the principle outlined above. The program was structured to allow a step-wise progression in skills development as cognitive aspects were acquired. Faculty developed the program during three general retreats, one for each year of the program. The goals of the retreats were to determine the clinical competencies required of a newly graduated mixed animal practitioner, and to develop a list of skills that would be essential for this individual to be able to perform on their first day of practice. Retreat participants included pre-clinical and clinical faculty, as well as members of the DVLC. Similar skills were then arranged into groups in order to start developing a framework for the laboratory sessions. The overarching goals of the clinical skills program at the University of Calgary are listed in table 1.

Once the goals were identified, we then moved into the instructional design phase. The concept of "nested skill set teaching" was developed, whereby each clinical procedure/skill that a student should be able to perform at the end of the DVM program was broken down into its respective components. Simple skills were then purposefully taught during the first year and subsequent components of the skill or procedure were taught in later sessions within the same year, or over the following

*Corresponding author: Emma K. Read, Assistant Professor Veterinary Medica Education, Faculty of Veterinary Medicine, Department of Veterinary Clinical and Diagnostic Sciences, University of Calgary, 3330 Hospital Drive NW Calgary, Alberta, T3H 4N1, Canada, Tel: 403-210-6250; Fax: 403-239-6984; E-mail ekread@ucalgary.ca

Received March 28, 2013; Accepted May 06, 2013; Published May 08, 2013

Citation: Read EK, Hecker KG (2013) The Development and Delivery of a Systematic Veterinary Clisnical Skills Education Program at the University of Calgary. J Veterinar Sci Technol S4: 004. doi:10.4172/2157-7579.S4-004

Copyright: @ 2013 Read EK, et al. This is an open-access article distributed under the terms of the Creative Commons Attribution License, which permits unrestricted use, distribution, and reproduction in any medium, provided the original author and source are credited. 
The clinical skills course will:

Provide global skill proficiency not specific technical knowledge to ensure a framework of skills and knowledge on which to draw from

- $\quad$ Provide a systematic approach to teaching skills (nested skills set teaching and essential skills lists)

- Provide a context and create enthusiasm for basic and theoretical knowledge

- Allow development of skills with feedback from experts

- Create opportunities for self-learning and dedicated skills practice using live animals and simulation

Table 1: Overarching goals of the clinical skills program.

years. This integration of skills development was designed to provide learners the opportunity to acquire skills in a systematic manner that emphasized repetition, practice, and the explicit addition of skills to those previously learned. In human medicine, psychomotor skills have been classified along a continuum from reproductive to productive [3]. Reproductive skills are those that are applied in a stable environment, that are repetitive in nature, that follow a uniform script, and that are learned by "rote learning" i.e., tying a surgical knot. Productive skills are more complex because they incorporate subsets of reproductive skills while also requiring strategic thinking. Productive skills call for a deeper understanding of the underlying principles to inform decisionmaking. An example of this would be the performance of a surgical procedure while incorporating the principle of asepsis [3]. Eventually, productive skills are performed in an unpredictable environment, the clinical setting, and students benefit from learning the skills across a wide range of case examples. Moving from demonstrating reproductive skills to performing productive skills is the difference between "shows how" and "does" on Miller's professional pyramid [4], and has been a goal of the UCVM clinical skills program since its inception. A stepwise progression of skills training and knowledge ensures learner confidence while providing opportunity for expert feedback and refinement of learner abilities.

Three levels of technical competence in medical professionals have been reported: familiarity, technical competence, and procedural competence [5]. Familiarity occurs when the learner can describe the indications for, the contraindications for, the procedural steps involved, and the adverse outcomes of the procedure. Technical competence occurs when the learner is able to actually perform the technical skills steps in the correct order in a simulated environment, in addition to describing the indications, contraindications and adverse outcomes. Procedural competence occurs when the leaner can perform the skill in the correct order without assistance or supervision on a live patient. In addition, they have the ability to assess the patient for indications and contraindications, can obtain informed consent, and can identify actual or potential complications [5].

As an example, in the surgical skills set, a first year student is taught to handle and use basic surgical instruments, tie simple, square, and surgeon's knots, tie simple skin suture patterns, clip and surgically prepare an animal, don a sterile gown and apply sterile gloves, and drape a surgical field. A second year student then integrates these introductory skills in a single lab early in the second year where they are required to surgically prepare a simulated patient, open a sterile surgical pack and prepare the instrumentation, surgically scrub their hands before applying a sterile gown and gloves, and draping the simulator. The second year learner then proceeds to make an incision in a simulated patient and performs a resection-anastamosis exercise on a cadaver equine gastrointestinal tract that further teaches them tissue handling and inverting suturing patterns. The progression of skills continues to later in the second year when the learner performs an exploratory abdominal surgery and cystotomy on a live swine model, which is anesthetized for them by a member of the technical team. In this manner, they incorporate all of the skills that they have learned to date and acquire new skills regarding the principles of tissue handling, hemostasis, inverting suture patterns, and abdominal wall closure. Finally, in the third year of the program students have dedicated training in anesthesia for a wide variety of species including dogs, cats, birds, horses, cattle and wildlife. This occurs before they are provided the opportunity to incorporate their anesthesia skills and surgery skills in live animal surgery exercises. In the third year of the program, each student will perform a castration on a dog, a cat, and a horse, an ovariohysterectomy on a cat and a dog, and an exploratory laparotomy on a cow. The student then enters the final year of the program in the DVLC and has the opportunity to refine their skills under the guidance of UCVM clinical faculty and DVLC practitioners, allowing them to move towards procedural competence upon graduation. Oversight of animal care and use in the course was provided by the veterinary sciences animal care committee in accordance with the principles of the Canadian Council on Animal Care [6].

\section{Creation of essential skills lists}

A comprehensive list of all skills was created after the three faculty retreats were completed. The skills were then arranged into a progressive series as described above. These lists created a blueprint for development of the course and allowed cataloging and cross-referencing of the skills. This was then provided to the DVLC practitioners for input on the skills taught before entry into the final year of the DVM program. These essential skills lists were also provided to the learners to create an expectation of responsibility for their own learning. Students were made aware that their future employers would expect them to be proficient in the skills listed in these documents and any of the skills listed were those that may be assessed. An example of the first year essential skills list is provided in Appendix 1.

\section{Structure of clinical skills sessions and associated learning activities}

In these courses, the lab sessions are each delivered as 3-hour blocks. There are 2 blocks for a given year of the course, on each of three weekdays. Each year has approximately 40 lab sessions. Each lab session typically includes a brief introductory lecture or refresher of previously taught skills at the beginning, followed by a demonstration of new skills and then time allowed for students to practice these skills with feedback from experts. A clinical simulation lab and animal interaction program have also been established to encourage students to spend time in selfdirected activities practicing their skills. This concept is supported by a recent meta-analysis showing that simulation-based medical education with deliberate practice yielded superior results compared to traditional clinical education [7]. In our program, the introduction of a dedicated practice lab with teaching materials and a simulation model to learn abdominocentesis in horses resulted in a $10 \%$ increase in mean score on an objective structured clinical examination of second year students. Similar improvement was not noted for other skills that were unavailable in the practice lab. It has been demonstrated that there is little increase in the learning between low and high fidelity simulators, therefore justifying the use of practical and feasible simulators to learn skills in this manner [8].

Near-peer teaching has been used to facilitate the learning of physical examination skills. Third year students were provided with a session outlining the principles of skills training including structuring a learning session appropriately, as well as providing feedback and evaluating learner styles and progression. Third year students were then 
asked to create teaching materials, develop a lesson plan and review physical examination skills with first year students. Development of this exercise resulted in a $20 \%$ improvement in OSCE performance of these skills within a single year.

Novel approaches have been created to provide more focused learning in some identified underserved areas. For instance, faculty perceived that many of the clinical problems in exotics practice stem from poor husbandry, therefore it was decided that all students should have mandatory training in small exotic animal care, handling, and feeding. Discussion amongst the faculty identified challenges in teaching skills and knowledge in a number of species in a meaningful manner within the confines of individual lab sessions, therefore a take home assignment has been created with the goal of providing first hand experience in the care of these animals. In the second year of the program, a group of small exotic mammals has been procured and assigned to go home with students on a rotating basis. Students are responsible for feeding, cleaning, and caring for the animals, as well as for maintaining a daily log of the animal's progress and activities. A similar lab session in the third year of the program is focused on reptiles and is a cooperative venture with a local biological sciences vivarium. The reptiles are loaned to the veterinary school and are housed on campus. Following an introductory lab session, students care for the animals and become familiar with their dietary needs, housing, thermal requirements, and physical exam characteristics, which they are required to record in a daily log. Specialists in the DVLC and on faculty provided an initial session to teach the basics of handling, physical examination, and caring for the animals, and provide on-going consultation as the project progresses. Students participate in resolving any health or management issues that arise in the individual animals.

\section{Creation and use of simulators}

Simulation is used in the program not to replace live animal use, but to allow learners to develop skills and confidence prior to transferring those skills to live animals. This avoids detrimental animal usage and allows for greater practice opportunities. The use of simulators has been embraced at UCVM in consideration of Russell and Burch's 3 R's of animal use- replacement, reduction and refinement [9].

Students are first taught and progress from low to high fidelity simulators, and then to live animals. In the same session or in a subsequent session, they are then required to perform the same skill on a live animal [10]. Simple simulators have been constructed from readily available supplies and are used to teach the skill basics (e.g., rubber latex tubing attached to a fluid bag filled with red food dyed water, mounted to a $2 \times 4$ ' piece of wood and covered in calf hide serves to make a model for teaching venipuncture and catheter placement). We have also developed sophisticated simulators as needed including a "colic horse" model and a "bovine dystocia" model. The "colic horse" is useful for teaching abdominocentesis, introductory rectal palpation, and 3-dimensional anatomical thinking. Through placement of a cadaver and then simulated uterus, and ovaries in this model, we have also taught the skills of uterine biopsy/culture and artificial insemination. The "bovine dystocia" model has a functional udder with 4 separate quarters that can be used for teaching milk sampling and evaluation, as well as mammary care and cleaning. The simulator also consists of a cow and calf component that allows for teaching normal delivery, fetotomy, and dystocia correction skills (Figures 1 and 2) [10].

Simulated clinical scenarios have also been developed to help teach these courses. For instance, a horse rescue scenario was created using an overturned horse trailer with a model horse inside. The student learners

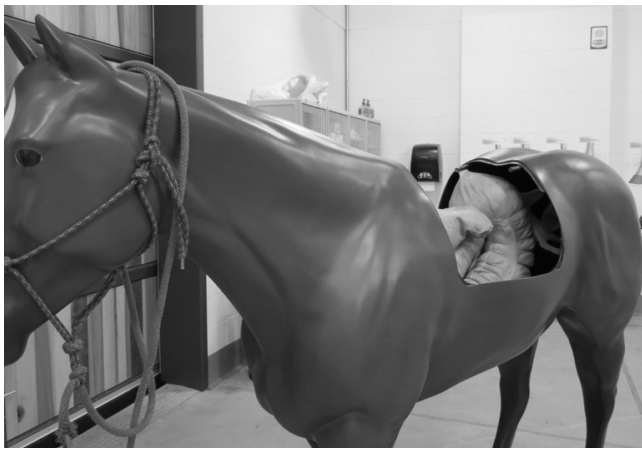

Figure 1: The "colic horse" model used to teach abdominocentesis, introductory rectal palpation, and 3-dimensional anatomical thinking.

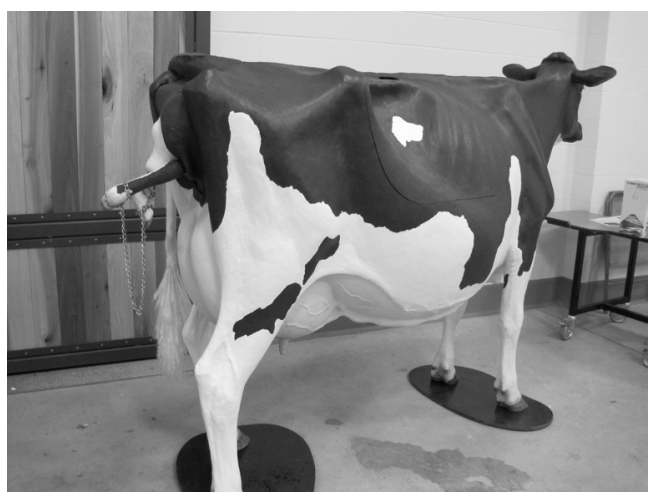

Figure 2: The "bovine dystocia" model with a cow and calf component that allows for teaching normal delivery, fetotomy and dystocia correction skills.

are asked to work as a team to rescue the trapped horse. A truck stocked with veterinary supplies is provided to allow students to complete the task. The scenario lasts 45 minutes and is conducted without instructor input. Immediately afterwards, students are debriefed using photographs and videos of their performance to create a dialogue, centered around components of the scenario that were handled correctly and areas that could be improved in their next encounter with a large animal in distress. This session incorporates many aspects of basic veterinary knowledge such as fracture management, pharmaceutical intervention, fluid administration, animal restraint, client communication, ethics, and jurisprudence.

\section{Live animal procurement for surgical exercises}

Working in conjunction with our partners in the DVLC, the faculty created a novel method to procure animals for the surgery labs. Practitioners in the DVLC are asked to help identify clients in need of low-cost surgery service. The clients are required to pay their veterinarian a nominal fee and the veterinarian then refers the client to the UCVM. The students receive the animal at the Clinical Skills Building, perform a physical examination and bloodwork preoperatively, spay or neuter the animal and care for it post-operatively before discharging it into the care of the client. The UCVM does not receive any payment from the client for services rendered and the client returns to the referring veterinarian for any required after care. Should follow-up care be needed, UCVM assumes the cost of any complications related directly to the surgery and the DVLC partner alerts the clinical skills faculty so that follow-up can be completed with the student surgery team at the practice. This program is novel because 


\section{Advantages}

- Indicates areas where teaching could be improved (when students consistently falter at the same point).

- Removes the "halo" effect and results in increasingly objective measurement of student performance (increased reliability) [13].

- Students accept format as "real life" and want to succeed-increased student acceptance of assessment (face validity) [13].

- Tests technical skill competence (tests "shows how" or "does") [4].

- Team building amongst faculty, DVLC and staff.

- Increases DVLC engagement and interest in program.

\section{Disadvantages}

- A learning curve exists to writing and conducting these exams.

Scenarios are contrived in most cases to help standardize across students and allow testing in this format-not all skills taught may be testable using this format and productive skills may not be properly assessed [13].

It is an indirect test of the knowledge component of skills training.

Faculty, staff intensive.

Resource intensive (supplies, animals, space) [13]

- Challenging to administer with a large student body.

Table 2: Advantages and disadvantages of using objective structured clinical examination (OSCE).

the provision of case material is entirely furnished via the goodwill of the practitioners and the veterinary school does not compete for cases with the local practices.

\section{Physical Infrastructure}

The purpose-built 80000 square foot Clinical Skills Building (CSB) was completed in 2009. The use of the building has allowed for offering multiple lab sessions on various species at the same time, dedication of space to create a simulation practice lab, and the freedom to teach the program without other conflicting demands on time, space or access to technical staff. The small animal facility houses a surgical facility and a communication skills laboratory with examination rooms complete with one-way mirrors, video cameras, and microphones for training students with simulated client interactions. The equine facility incorporates a surgical suite and recovery room, a series of stalls, and a stocks area that can accommodate up to five horses at one time. The bovine facility houses custom-built headgates that convert into individual animal holding pens, a handling system, and hydraulic tilt tables. There is also a feedlot style collection system outside to teach the students cattle processing skills. Also housed within the CSB is the anatomy lab, the gross pathology lab, and a diagnostic pathology center. A clinical simulation lab space has also been created in the building and is available to students at any time to practice the skills they have been taught.

\section{Assessment of student performance}

Development of student assessment methods: This course is delivered in a hands-on manner and is designed to teach practical skills. Objective Structured Clinical Evaluations (OSCE's) are used to assess student performance in all 3 years to ensure testing of hands-on abilities. The OSCE's are a series of timed stations that vary in duration according to the difficulty level of the skill, and the year of the course. All of the OSCE stations feature a standardized marking sheet of checklist items that are assessed as "yes/no" responses that indicate performance or non-performance of that element of the skill. All OSCE's have a predetermined minimum performance level that is adhered to in grading. The use of OSCE's has been shown to reduce examiner variability and increase reliability [11]. Some of the advantages and disadvantages of the OSCEs are shown in table 2.

In our program, year one OSCE stations are simple 5-minute reproductive skill assessments [12]. In year two, we use a series of stations that move the learner through history taking, to physical examination, to problem list generation and finally, diagnostic testing. A typical second year OSCE incorporates three different species and is $12-15$ stations in length with each station being 8 minutes duration. In year three, we attempt to incorporate clinical reasoning and critical thinking, as well as practical skills using 20-minute stations that contain multiple facets of a single clinical case. This integration is designed to test procedural competence across one case and is conducted at the individual's own pace within the time allotted. As an example, the candidate would be asked to manage an anesthesthetic complication by progressing from recognition of the problem, to requesting further diagnostic testing, to taking corrective action to treat the problem.

For each OSCE station, checklists are created based upon the skills that were taught and the objectives that were outlined during the skills session. Minimum performance levels (MPLs) for each station are created using a modified Ebel method, a priori [13].

\section{Conclusion}

In conclusion, the clinical skills course provides students guided facilitation in practical skills development starting in their first year and building sequentially through to the final year of their training. This novel three-year program has been purpose built, to ensure, through teaching and evaluation, that students have foundational skills before entering their clinical rotations in the DVLC.

\section{References}

1. Michels ME, Evans DE, Blok GA (2012) What is a clinical skill? Searching for order in chaos through a modified Delphi process. Med Teach 34: e573-581.

2. Smeak DD (2007) Teaching surgery to the veterinary novice: The Ohio State University Experience. J Vet Med Educ 34: 620-627.

3. Acton RD, Chipman JG, Gilkeson J, Schmitz CC (2010) Synthesis versus imitation: Evaluation of a medical student simulation curriculum via objective structured assessment of technical skill. J Surg Educ 67: 173-178.

4. Miller GE (1990) The assessment of clinical skills/competence/performance. Acad Med 65: S63-67.

5. Raninger C (2012) A comprehensive procedural skills curriculum for medical students: Skills selection and curricular integration (Abstract-12 $2^{\text {th }}$ Annual International Meeting on Simulation in Healthcare, January 2012). Simulation in Healthcare 6: 507 .

6. http://www.ccac.ca/en_/education/niaut/stream/cs-3rs

7. McGaghie WC, Issenberg SB, Cohen ER, Barsuk JH, Wayne DB (2011) Does Simulation-Based Medical Education with Deliberate Practice Yield Better Results Than Traditional Clinical Education? A Meta-analytic Comparative Review of the Evidence. Acad Med 86: 706-711.

8. Norman G, Dore K, Grierson L (2012) The minimal relationship between simulation fidelity and the transfer of learning. Med Educ 46: 636-647.

9. Russell WMS, Burch RL (1959) The Principles of Humane Experimenta Technique. Methuen, London.

10. Brydges R, Carnahan H, Rose D, Rose L, Dubrowski A (2010) Coordinating progressive levels of simulation fidelity to maximize educational benefit. Acad Med 85: 806-812.

11. May SA, Head SD (2010) Assessment of technical skills: Best practices. J Vet Med Educ 37: 258-265.

12. Hecker K, Read EK, Vallevand A, Krebs G, Donszelmann D, et al. (2010) Assessment of First-Year Veterinary Students' Clinical Skills Using Objective Structured Clinical Examinations. J Vet Med Educ 37: 395-402.

13. Ebel RL, Frisbie DA (1991) Essentials of Educational Measurement. (4thedn) Prentice Hall, Toronto, Canada. 
Citation: Read EK, Hecker KG (2013) The Development and Delivery of a Systematic Veterinary Clinical Skills Education Program at the University of Calgary. J Veterinar Sci Technol S4: 004. doi:10.4172/2157-7579.S4-004

\begin{tabular}{|c|c|}
\hline Skill area & Description of the skills \\
\hline \multirow[t]{2}{*}{ Biosecurity and Biosafety } & $\begin{array}{l}\text { Applying personal protective clothing and equipment in a variety of veterinary settings (pathology, swine/poultry barn, large animal and } \\
\text { small animal handling) }\end{array}$ \\
\hline & Hand washing; instrument cleaning; facility cleaning; animal movement \\
\hline \multirow{7}{*}{ Bovine } & Distant examination; manipulation of animal movement (using flight zone and point of balance). \\
\hline & $\begin{array}{l}\text { Application of stock person's terminology; knowledge of the production cycle of a beef animal/dairy animal; safe use of bovine handling } \\
\text { facilities (headgates, alleys, tubs); Husbandry practices (castration, ear tagging, dehorning, auricular subcutaneous injection, tag reading } \\
\text { and identification). }\end{array}$ \\
\hline & Safe handling practices (catching, haltering, restraint techniques, use of tilt tables) \\
\hline & Evaluation of production records. \\
\hline & $\begin{array}{l}\text { Physical examination; history taking (individual animal and herd); conformation assessment and evaluation; udder evaluation and milk } \\
\text { sample collection; California Mastitis test; blood and urine sampling. }\end{array}$ \\
\hline & Foot trimming; gait analysis. \\
\hline & Palpation of and identification of cadaver reproductive tract; retraction of cadaver uterus; reproductive examination of the cow and bull. \\
\hline Clinical Pathology & $\begin{array}{l}\text { Collection of blood; creation and evaluation of blood smears; determination of PCV/TS; evaluation of the blood cells; urinalysis, species } \\
\text { comparisons for urine and blood samples. }\end{array}$ \\
\hline \multirow{6}{*}{ Equine } & Horsemanship skills (behavior, manipulation of animal movement); horseman's terminology; knowledge of disciplines and uses. \\
\hline & Safe handling practices (haltering, leading, tying, picking up limbs, distraction techniques, restraint techniques, injection sites). \\
\hline & Physical examination; history taking; conformation assessment and evaluation; record keeping. \\
\hline & Bandaging; passport and Coggin's form completion; estimation of height and weight; body condition scoring. \\
\hline & Reproductive exam of the mare's external genitalia, vaginal palpation and speculum examination. \\
\hline & Foot care: Hoof tester application, foot trim, remove shoe, safe and proper use of farrier tools, identification of shoeing basics. \\
\hline Necropsy & $\begin{array}{l}\text { Proper technique for monogastric animal necropsy (open } 3 \text { body cavities, remove organs and evaluate); Safe knife and instrument } \\
\text { handling; biosafety. }\end{array}$ \\
\hline Pocket Pets & $\begin{array}{l}\text { Handling and restraint of rats, mice, rabbits, guinea pigs, hamsters, gerbils, budgies; venipuncture techniques; intramuscular and } \\
\text { intravenous injections; euthanasia; husbandry requirements. }\end{array}$ \\
\hline Poultry and Avian & $\begin{array}{l}\text { Handling and restraining; physical examination, blood collection; humane euthanasia; postmortem examination; injection techniques } \\
\text { including vaccination. }\end{array}$ \\
\hline \multirow{3}{*}{ Radiology } & Safety practices (equipment, labeling and storage, machine settings and use, restraint, legal requirements). \\
\hline & Equipment knowledge and use (plain film vs. CR vs. DR). \\
\hline & Patient positioning and restraint, terminology. \\
\hline \multirow{5}{*}{ Small Animal } & Restraint and handling of dogs and cats, microchip identification and reading, nail trimming, medication administration (PO, SQ, IM, IV). \\
\hline & $\begin{array}{l}\text { Physical examination; history taking; conformation assessment and evaluation; record keeping; medication storage and labeling; } \\
\text { calculation of nutritional requirements. }\end{array}$ \\
\hline & Venipuncture and catheter placement on simulated patient. \\
\hline & Musculoskeletal examination (gait, range of motion, palpation); Basic bandaging; Splint application. \\
\hline & Reproductive examination (vaginal cytology). \\
\hline $\begin{array}{l}\text { Small Ruminant and } \\
\text { Camelids }\end{array}$ & $\begin{array}{l}\text { Restraint and handling; injection sites; husbandry practices; use of laymen's terminology; physical examination; transcutaneous } \\
\text { pregnancy ultrasound examination; ram breeding soundness exam. }\end{array}$ \\
\hline \multirow{6}{*}{ Surgery } & $\begin{array}{l}\text { Differentiation of basic instrumentation (needle drivers, scissors, forceps, scalpel blades and handles); Basic instrument handling and } \\
\text { dissection techniques }\end{array}$ \\
\hline & Knot tying (simple, square, surgeons). \\
\hline & Suturing (simple interrupted and simple continuous pattern). \\
\hline & Surgical preparation of the patient; handscrubbing; gowning; open and closed gloving; draping. \\
\hline & Skin suturing patterns (cruciate, horizontal mattress, vertical mattress, far-near-near-far, Ford interlocking, intradermal). \\
\hline & Differentiation of: Needle size/type, Suture material size and type, Tissue types and layers. \\
\hline Swine & Restraint and movement of swine; blood collection; facility design and safe use. \\
\hline Ultrasonography & Machine use; image interpretation basics; identification of structures in cadavers and simulators. \\
\hline Wildlife and Game Farm & Safe handling of common game farm species; handling facility design and assessment; common husbandry practice. \\
\hline
\end{tabular}

